\title{
Guest editorial: Information reuse, integration, and reusable systems
}

\author{
Chengcui Zhang • Elisa Bertino • \\ Bhavani Thuraisingham • James Joshi
}

Published online: 26 September 2014

(C) Springer Science+Business Media New York 2014

\section{Introduction}

Given the emerging global Information-centric IT landscape that has tremendous social and economic implications, effectively processing and integrating huge volumes of information from diverse sources to enable effective decision making and knowledge generation have become one of the most significant challenges of current times. This special issue intends to publish high quality papers that present novel techniques and frameworks that maximize the reuse of information by creating simple, rich, and reusable knowledge representations and consequently explore strategies for integrating this knowledge into systems and applications. The emphasis of this special issue is subdivided into three major tracks: information reuse, information integration, and reusable systems. Information reuse addresses the theory and practice of representation optimization, with the goal to minimize the time and effort spent to develop new information technologies, architectures, frameworks, and repositories, etc. Possibilities of reusing existing resources, such as data/software repositories, should be exploited to avoid waste of effort and resources in

C. Zhang $(\bowtie)$

The University of Alabama at Birmingham, Birmingham, USA

e-mail: zhang@cis.uab.edu

E. Bertino

Purdue University, West Lafayette, USA

e-mail: bertino@purdue.edu

B. Thuraisingham

The University of Texas at Dallas, Richardson, USA

e-mail: bhavani.thuraisingham@utdallas.edu

J. Joshi

University of Pittsburge, Pittsburgh, USA

e-mail: jjoshi@pitt.edu developing new products. Information integration focuses on innovative strategies and algorithms for applying integration approaches in novel domains. Information reuse and integration are essential in avoiding duplicate efforts. Reusable systems focuses on deploying models and corresponding processes, which enable Information Reuse and Integration to play a pivotal role in enhancing decisionmaking processes in various application domains.

Despite the progress the field has seen in recent years, many challenges still stand and new ones are emerging. One recent major challenge with information reuse and integration in big data analysis is attributed to incompatible data models, lack of effective and efficient filtering of information from high volumes of data from distributed sources for decision making, varying information quality (e.g., accuracy and timeliness) and granularity, security and privacy concerns about data protection which prevents taking full advantage of the data, and complex interdependence across heterogeneous computing resources. Information systems need to evolve to adapt to new technologies and needs, however, the major challenge lies in selecting the components to be reused, and how to maximize their compatibility with the exiting and the new components to be developed. Some of the risks include incompatible architectural approaches, difficult and expensive to verify whether the reusable components meet the qualify/ security requirements of the evolved system, and the expense to plug in reusable components.

\section{About this special issue}

This special issue consists of the best papers from an open call selected on a competitive basis and invited papers that are extended versions of selected papers accepted at the 13th IEEE International Conference on Information Reuse and Integration (IRI'12, Las Vegas, Nevada, USA, August 8-10, 
2012.) IRI aims at maximizing the reuse of information by creating simple, rich, and reusable knowledge representations and consequently explores strategies for integrating this knowledge into legacy systems. IRI plays a pivotal role in the capture, representation, maintenance, integration, validation, and extrapolation of information; and applies both information and knowledge for enhancing decision-making in various application domains. During more than a decade of conferences, IRI has established itself as an internationally renowned forum for researchers and practitioners to exchange ideas, connect with colleagues, and advance the state of the art and practice of current and future research in information reuse and integration. The technical program of IRI 2012 consists of invited talks, full and short paper presentations, workshops, and panel discussions. The authors of invited IRI'12 papers were asked to revise their original IRI 2012 presentation and extend it by at least $30 \%$ new content. Each submission was rigorously reviewed by a panel of referees selected from the conference program committee and a selected few external reviewers.

The papers included in this special issue address original research in information filtering and integration, multi-model collaboration, feature selection, association mining, and reusable software systems, and propose novel methods for applications such as web information extraction, story chain discovery in newswire articles, video semantic concept mining, software defect prediction, and code clone aspectualization.

The paper, "Finding story chains in newswire articles using random walks" by Zhu and Oates (2014), proposes an efficient and effective algorithm to automatically find a chain of news articles that reveal hidden relationships among different events and various aspects of a breaking news event. When a breaking news event occurs, many news media rush to report the same event, often with highly similar news content. Traditional keyword-based search neither provides support for chaining relevant events together, nor does it eliminate duplicate content. The algorithm presented in this paper uses the idea of random walks to piece relevant information together and at that same time automatically removes redundant articles from the story chain in an efficient manner. Their results show that this algorithm can produce coherent story chains without much redundancy.

While the abovementioned paper aims at integrating information from different web resources (e.g., news articles collected from the webpages of various media outlets), the paper, "An FAR-SW based approach for webpage information extraction" by Zhan et al. (2014) targets the problem of automated extraction of target content (e.g., news articles) from a webpage, which is not a trivial task given the amount of nonmain-text objects presented on a webpage such as logos, ad blocks, and dynamic navigation menus. The approach proposed in this paper is statistics-based and incorporates fuzzy association rule (FAR) to discover the common patterns/ features main text lines exhibit on a webpage. A preprocessing step is carried to extract various content and layout features of main text lines, and a supervised learning is conducted to summarize the robust fuzzy association rules discovered from the training main text lines. Those rules will then be applied to a previously unseen webpage to extract candidate main text lines which are later grouped into candidate main text blocks. Candidate blocks are then subject to a selection process in order to form the final detected main text regions for that webpage. Their experimental results show that their approach outperforms two other well-known approaches, the Document Object Model (DOM)-based and the Visionbased approaches.

Another paper, "Concept-concept association information integration and multi-model collaboration for multimedia semantic concept detection" by Meng and Shyu (2014), proposes a method for deep exploration of concept-concept relationship and information integration to achieve more accurate video concept detection. First, by using existing video semantic concept detectors, the concepts associated with a video shot are detected and scored. Next the associations between and among extracted video concepts (input as labels and scores) are explored using a logistic regression model and a collaboration model, and are further subject to a re-ranking mechanism that takes advantage of such concept-concept associations to help improve the video concept detection. Their experimental results demonstrate that with the deep exploration of concept associations and information integration, the detection of high-level concepts from video shots can be further improved by a non-trivial margin.

Khoshgoftaar et al. (2014) target the problem of feature selection in a high dimensional feature space and conduct a comparative study of various feature selection techniques for software defect prediction in their paper "A comparative study of iterative and non-iterative feature selection techniques for software defect prediction." In particular, they try to tackle the problems of feature selection and imbalanced training data concurrently by using an iterative feature selection approach that iteratively does data sampling (to address the class imbalance issue) and feature selection (to address the curse of dimensionality issue). The final set of selected features is the combined ranked list of ranked features from individual iterations of sampling and feature selection. In total 18 different feature selections algorithms are tested. For fair comparison, they also test the non-iterative approach and compare its performance with the iterative one. Real software systems are collected and tested, with different levels of class imbalance. They find that the iterative feature selection approach outperforms the non-iterative one in almost all cases.

Another more commonly adopted way to detect software defect is through the use of software test cases. However, the generation of test cases has been considered one of the most challenging tasks in test software systems. Model checkers 
have been reused to assist in generating test cases not only in a more efficient way, but also in a more powerful way in the sense that a model checker can potentially enable systematic search of the state space of a software system and the exploration of test cases that are not so obvious even to experienced software developers or quality engineers. This paper, "A unified framework for evaluating test criteria in modelchecking-assisted test case generation" by Zeng and Tan (2014) describes a unified framework that evaluates various test criteria and the efficiency of model-checking-assisted test case generation. An automated test case generation environment has been developed and described in great details in this paper, which, together with the consistent comparison of various test criteria, provides practitioners with valuable insights and suggestions on how to integrate model-checkingassisted test case generation into their testing process.

Aiming at the problem of code clones harming the maintainability and reusability of codes, Samuel et al. (2014) propose to use Aspect Oriented Programming (AOP) to encapsulate code clones in a special kind of programming construct called aspects in their paper "Aspectualization of code clones - an algorithmic approach." Encapsulating code clones this way reduces code scattering and inconsistent changes thereby reducing the risk of system fault and increasing the reusability of the code. Their proposed approach scales to large and complex software that involves substantial overlapping of cross-cutting concerns represented in the form of scattered code clones. The results show that the recomposed code using aspects performs as well as the original with code clones and executes even faster. All the four method typed clones can be successfully handled by the proposed algorithmic approach called CC2ASPECT.

This paper, "Measuring the performance of aspect oriented software: A case study of Leader/Followers and Half-Sync/ Half-Async architectures" by Lung et al. (2014), further measures the impact of AOP on software performance by comparing the performance of the aspect-composed code with that of the original code. In particular, they reverse-engineers two well-known concurrency architectures, the so called Leader/ Followers and Half-Sync/Half-Async architectures, encapsulates cross-cutting concerns in aspects, and evaluates the impact of AOP incurred overhead (e.g., control flow switching, increased number of join points) on the code performance. According to their experimental results, AOP overhead does not have any significant impact on code performance, and sometimes aspect-composed code even performs better than the original code. They further study the impact of cache fault rate on the performance for both aspect-composed and nonaspect codes. Again no significant impact of AOP overhead is observed, but they do conclude that there is a close correlation between cache fault rate and performance which may favor aspect-composed code if those aspects are frequently accessed.
Acknowledgments The guest editors wish to thank all of the authors, reviewers and publisher. Without their excellent work, and the devotion of their valuable time, this special issue would not have been possible.

Referees for this special issue:

Zhan $\mathrm{Bu}$

Min Chen

Zekai Demirezen

Song Gao

Jeff Gray

Rajan Kharel

Qichao Liu

Nan Niu

Gordon Novak

Mourad Oussallah

Richa Tiwari

Chengcui Zhang

John Zhang

\section{References}

Khoshgoftaar, T. M., Gao, K., Napolitano, A., \& Wald, R. (2014). A comparative study of iterative and non-iterative feature selection techniques for software defect prediction. Information Systems Frontiers, 16(5). doi:10.1007/s10796-013-9430-0.

Lung, C.-H., Samuel, A. A., \& Liu, W.-L. (2014). Measuring the performance of aspect oriented software: A case study of Leader/Followers and Half-Sync/Half-Async architectures. Information Systems Frontiers, 16(5). doi:10.1007/s10796013-9423-z.

Meng, T. \& Shyu, M.-L. (2014). Concept-concept association information integration and multi-model collaboration for multimedia semantic concept detection. Information Systems Frontiers, 16(5). doi: 10.1007/s10796-013-9427-8.

Samuel, A. A., Gakhar, A. S., \& Lung, C.-H. (2014). Aspectualization of code clones - an algorithmic approach. Information Systems Frontiers, 16(5). doi:10.1007/s10796-013-9428-7.

Zeng, B. \& Tan, L. (2014). A unified framework for evaluating test criteria in model-checking-assisted test case generation. Information Systems Frontiers, 16(5). doi:10.1007/s10796-0139424-y.

Zhan, B., Zhang, C., Xia, Z., \& Wang, J. (2014). An FAR-SW based approach for webpage information extraction. Information Systems Frontiers, 16(5). doi:10.1007/s10796-013-9412-2.

Zhu, X. \& Oates, T. (2014). Finding story chains in newswire articles using random walks. Information Systems Frontiers, 16(5). doi:10. 1007/s10796-013-9420-2.

Chengcui Zhang Is an Associate Professor in the Department of Computer and Information Sciences, the University of Alabama at Birmingham and an Associate Director of the Knowledge Discovery and Data Mining Laboratory. She has authored and coauthored over 130 research papers focusing on Multimedia Data Mining, Multimedia Information Retrieval, Multimedia Databases, Bioinformatics, and Geographic Information Systems. She has served on more than 70 international conferences and workshops and taken a leadership role at the 2014 I.E. International Conference on Multimedia and Expo, the 2012-2013 I.E. International Conference on Information Reuse and Integration, 2012 I.E. International Symposium on Multimedia, and 2010 I.E. International Conference on Multimedia and Ubiquitous Engineering (MUE). In addition, she is a frequent panelist for National Science Foundation (NSF) panels and an Editorial Board member of several international journals. 
Elisa Bertino Is professor of computer science at Purdue University, and serves as Director of Purdue Cyber Center and Research Director of the Center for Information and Research in Information Assurance and Security (CERIAS). Prior to joining Purdue in 2004, she was a professor and department head at the Department of Computer Science and Communication of the University of Milan. She has been a visiting researcher at the IBM Research Laboratory (now Almaden) in San Jose, at the Microelectronics and Computer Technology Corporation, at Rutgers University, at Telcordia Technologies. Her recent research focuses on database security, digital identity management, policy systems, and security for web services. She is a Fellow of ACM and of IEEE. She received the IEEE Computer Society 2002 Technical Achievement Award and the IEEE Computer Society 2005 Kanai Award. She is currently serving as EiC of IEEE Transactions on Dependable and Secure Computing.

Bhavani Thuraisingham Is a Louis A. Beecherl, Jr. Distinguished Professor and Director of the Cyber Security Research and Education Institute at The University of Texas at Dallas. She is an elected Fellow of IEEE, the AAAS, the British Computer Society, the SPDS (Society for Design and Process Science) and the Society of Information Reuse and Integration (subcommittee of IEEE Systems, Man and Cybernetics
Society). She has unique experience working in commercial industry, research laboratory, US government and academia and her 30+ year career includes research and development, technology transfer, product development, program management, and consulting for the federal government. Her work has resulted in $100+$ journal articles, $200+$ conference papers, three US patents, twelve books and numerous awards. Under her leadership, the institute has generated over \$25 million in research and education funding from AFOSR, NSF, IARPA, NGA, NASA, ONR, NIH, DARPA, Raytheon, IBM, Tektronix and others.

James Joshi Is an associate professor in the School of Information Sciences at the University of Pittsburgh. He is a co-founder and the director of the Laboratory of Education and Research on Security Assured Information Systems (LERSAIS). He received his MS in Computer Science and $\mathrm{PhD}$ in Computer Engineering degrees from Purdue University. His research interests include Access Control Models, Security and Privacy of Distributed Multimedia Systems, Trust Management and Information Survivability. He is a recipient of the NSF-CAREER award. $\mathrm{He}$ is a Fellow of the Society of Information Reuse and Integration and a senior member of the IEEE and ACM. He directs the NSF CyberCorp Scholarship for Service program at the University of Pittsburgh. 